\title{
Psychological Statuses of Premenopausal Women Before and After Hysterectomy
}

\author{
Yongmei $\mathrm{Li}^{1}$, Ni Wang ${ }^{2}$, Lin $\mathrm{Li}^{1}$, Jichang $\mathrm{Li}^{1}{ }^{1}$, \\ ${ }^{1}$ Department of Pathology, Binzhou People's Hospital, Binzhou City, China \\ ${ }^{2}$ Department of Ultrasound, Binzhou People's Hospital, Binzhou City, China
}

Email address:

Lijichang2017@163.com (Jichang Li)

${ }^{*}$ Corresponding author

\section{To cite this article:}

Yongmei Li, Ni Wang, Lin Li, Jichang Li. Psychological Statuses of Premenopausal Women Before and After Hysterectomy. Clinical Medicine Research. Vol. 6, No. 4, 2017, pp. 143-148. doi: 10.11648/j.cmr.20170604.16

Received: July 7, 2017; Accepted: July 21, 2017; Published: August 2, 2017

\begin{abstract}
Objective: This paper aims to explore the trend, extent and impacting factors of the change in the psychological status of women before and after hysterectomy. Methods: A prospective study was performed to investigate the psychological statuses of 98 female subjects who underwent hysterectomy upon admission and three months after the hysterectomy. Results: The self-rating of the psychological status survey showed significantly lower factor scores including the postoperative BDI and SCL-90 (except paranoid factors) scores than those before the surgery and the same HAMD score as the nurse-administered rating scale. The incidence rate of moderate to severe depression dropped from $48.98 \%$ to $13.54 \%$ after the surgery. Differently aged patients showed different change extents of SCL-90, and the postoperative BDI and HAMD scores were significantly positively correlated with the preoperative levels. The preoperative HAMD score was negatively correlated with the length of education and positively correlated with the chief complaint. The postoperative HAMD score was positively correlated with the parity and the presence of medical complications and negatively correlated with age. Conclusion: Hysterectomy benefits the psychiatric and somatic health of the patients, and the women who were younger, more prolific, less educated, complain of dysmenorrhea or menorrhagia and have other medical disorders are more prone to perioperative depression.
\end{abstract}

Keywords: Investigation, Hysterectomy, Psychological Status

\section{Introduction}

Hysterectomy is a very common gynecological surgery with approximately 50,000 women recipients each year in the United States alone. Since the uterus plays a special social, sexual, and psychological role, diseases and surgeries that are related with the uterus can bring forth very high incidence rate of psychiatric disorders [1, 2]. With the change in the socio-biomedical model, change of the psychological status of women before and after hysterectomy has become a focus of study in the psychosomatic medicine [3, 4]. In this paper, a prospective research method is used to analyze the trend, extent and impacting factors of the change in the psychological status of women before and after hysterectomy so as to find out the risk factors that may negatively affect the psychological status of the patients and identify the population susceptible to psychiatric disorders. This study will provide basis for the postoperative rehabilitation and mental health-related undertakings and safeguard the psychiatric and somatic health of the patients during the perioperative period.

\section{Subjects and Methods}

\subsection{Subjects}

A total of 98 premenopausal women who underwent hysterectomy due to benign diseases after their admission to our hospital from January 2012 to October 2012 were included in this survey. They were aged between 34 and 52 years (44.8 on average), and had lengths of education ranging from 5 to 15 years ( 8.27 on average). All patients were free from obvious organic brain disorder, intellectual disturbance, past history and family history of psychiatric disorder, and damage to important organs. They could understand and complete the self-rating scales on their own. The patients had 
no experience of major stressful life events over the past year and did not receive any surgical therapy in the previous six months.

\subsection{Investigation Methods}

The survey is conducted with psychology test questionnaires upon the patient admission and after three months after the surgery. Symptom Checklist (SCL-90), Beck Depression Inventory (BDI), self-rated Health Measurement Scale (SRHMS) and Self-Concept Scale were chosen for the psychological self-rating scales in the measurement tools. The 24-item Hamilton Depression Rating Scale (HAMD) was used as the nurse-administered rating scale. A professionally trained medical staff was arranged to rate all patients.

\subsection{General Information}

The 98 patients had a shortest disease course of three months and a longest disease course of 14 years. Among these patients, 52 of them complained of menorrhagia, 26 complained of dysmenorrhea, 16 complained of both symptoms and four complained of none. 13 patients had past history of surgery, 37 had mild medical complications, 72 underwent total hysterectomy, 26 underwent subtotal hysterectomy, 18 had uterine appendages on one side resected and 11 had uterine appendages on both sides resected.

\subsection{Evaluation Criteria on Depress Status}

The self-rating depression scale Beck Depression Inventory determines the extent of the depression state according to the BDI scores. A BDI score not higher than 4 suggests no depression or very minimal depression, a BDI score between 5 and 13 suggests mild depression, a BDI score between 14 and 20 suggests moderate depression, and a BDI score higher than 21 suggests severe depression [5].

The nurse-administered rating scale Hamilton Depression Rating Scale determines the extent of the depression according to the HAMD scores. An HAMD score $\leq 8$ suggests no depression, an HAMD score between 9 and 20 suggests mild depression, an HAMD score between 21 and 35 suggests moderate depression, and an HAMD score higher than 35 suggests severe depression. A patient would be diagnosed with depression if he/she had an HAMD score higher than 20 [6].

\subsection{Self-Rated Health Measurement Scale (SRHMS) and Self Concept Scale}

SRHMS can be used for the self-ratings from the physical, psychological, and social aspects (with three sub-scales) and nine dimensions. The SRHMS is suitable for the health status evaluation of the population over the age of 14 . The full marks for the physical, psychological, and social health sub-scales and the SRHMS are 170, 150, 120 and 440 respectively. The SRHMS has a test-retest reliability of 0.857 as well as high content validity. A better health status is indicated with a higher SRHMS score [7].

The Self Concept Scale had 10 self-scores. In addition to self-score and self-criticism, it has two dimensions, i.e., structural dimension (self-identity, self-satisfaction, self-action) and content dimension (physical self, moral and ethical self, psychological self, family self and social self). This scale showed high reliability and validity in its domestic use [8].

\subsection{Statistical Analysis}

The obtained data are expressed as mean \pm standard deviation $(\mathrm{x} \pm \mathrm{s})$. The significance comparison between different groups was done with paired t-test, and the relationship between HAMD scores and the influencing factors was subject to multiple regression analysis.

\section{Results}

The patients were divided into four age groups: patients $<40$ years old, patients between 40 and 45 years old, patients between 45 and 50 years old, and patients $\geq 50$ years old. Two patients were lost in the follow-up with a loss rate of $2.04 \%$.

\subsection{Depression Status Trends Before and After Surgery}

It was revealed in the BDI and HAMD score comparison in the patients of the four age groups before and after the surgery that the postoperative BDI and HAMD scores of patients of all ages decreased significantly from the preoperative levels $(\mathrm{P}<0.05)$.

Table 1. BDI and HAMD scores before and after surgery.

\begin{tabular}{llll}
\hline \multirow{2}{*}{ Age } & BDI score & HAMD score \\
\cline { 2 - 4 } & Before $(\mathbf{n = 9 8 )}$ & After $(\mathbf{n = 9 6 )}$ & Before $(\mathbf{n = 9 8 )}$ \\
\hline$<40$ & $13.67 \pm 4.23$ & $10.22 \pm 4.35^{*}$ & $15.32 \pm 4.15$ \\
$40 \sim<45$ & $13.24 \pm 3.53$ & $9.25 \pm 3.14^{*}$ & $14.35 \pm 5.01$ \\
$45 \sim 50$ & $11.25 \pm 4.24$ & $8.35 \pm 3.53^{*}$ & $14.92 \pm 4.15$ \\
$\geq 50$ & $12.45 \pm 4.73$ & $8.74 \pm 4.07^{*}$ & $13.82 \pm 3.89$ \\
Total & $12.63 \pm 4.84$ & $9.07 \pm 4.61^{*}$ & $10.85 \pm 4.56^{*}$ \\
\hline
\end{tabular}

Compared with the results before surgery, ${ }^{*} \mathrm{P}<0.05$.

\subsection{Depression Status Changing Extents Before and After Surgery}

According to BDI scoring criteria, the results showed evidently relieved depression in the patients after the surgery. Six patients had no depression or very minimal depression before the surgery, and this number increased to 43 after the surgery. The numbers of patients with mild depression before 
and after the surgery were 37 and 45 respectively. There were 55 patients with moderate to severe depression before the surgery and this number dropped to 8 after the surgery. The incidence rate of severe depression in the self-rating decreased from $56.12 \%$ to $8.34 \%$ after the surgery (Table 2).

Based on the HAMD assessment rules, the results showed that there were 11 patients without depression before the surgery and this number increased to 34 after the surgery. There were 39 and 49 patients with mild depression before and after the surgery; and the number of patients with moderate to severe depression was 48 before the surgery and this number decreased to only 13 after the surgery. According to the diagnostic criteria, a patient would be diagnosed with depression if he/she has an HAMD score higher than 20. This study shows that this group of patients had a preoperative depression incidence rate of $48.98 \%$ and the incidence rate decreased to $13.54 \%$ after the surgery. The incidence rate of depression decreased significantly after the surgery (Table 3 ).

Table 2. BDI grading before and after surgery.

\begin{tabular}{lllll}
\hline \multirow{2}{*}{$\begin{array}{l}\text { BDI } \\
\text { value }\end{array}$} & \multicolumn{2}{c}{ Before $(\mathbf{n = 9 8})$} & \multicolumn{3}{c}{ After $(\mathbf{n = 9 6 )}$} \\
\cline { 2 - 5 } Case No. & Percentage & Case No. & Percentage \\
\hline$\leq 4$ & 6 & $6.12 \%$ & 43 & $44.78 \%$ \\
$5 \sim 13$ & 37 & $37.76 \%$ & 45 & $46.88 \%$ \\
$14 \sim 20$ & 46 & $46.94 \%$ & 8 & $8.34 \%$ \\
$\geq 21$ & 9 & $9.18 \%$ & 0 & 0 \\
\hline
\end{tabular}

Table 3. HAMD grading before and after surgery.

\begin{tabular}{lllll}
\hline HAMD & \multicolumn{2}{l}{ Before $(\mathbf{n = 9 8})$} & \multicolumn{3}{l}{ After $(\mathbf{n = 9 6 )}$} \\
\cline { 2 - 5 } value & Case No. & Percentage & Case No. & Percentage \\
\hline$\leq 8$ & 11 & $11.22 \%$ & 34 & $35.42 \%$ \\
$9 \sim 20$ & 39 & $39.80 \%$ & 49 & $51.04 \%$ \\
$21 \sim 35$ & 42 & $42.86 \%$ & 12 & $12.50 \%$ \\
$>35$ & 6 & $6.12 \%$ & 1 & $1.04 \%$ \\
\hline
\end{tabular}

\subsection{Factors Influencing Depression Status}

The postoperative BDI score was highly significantly correlated with the preoperative score $(\mathrm{r}=0.8962, \mathrm{P}<0.01)$, and the postoperative HAMD score was highly significantly correlated with the preoperative HAMD score $(\mathrm{r}=0.8857$, $\mathrm{P}<0.01)$. In order to explore to what extent various social factors affect the preoperative and postoperative HAMD scores of the patients, multiple regression analysis was conducted between the HAMD score and various factors such as age, length of occurrence of disease, length of education, occupation, parity, main symptoms, past history of surgery, number of children, the presence of medical complications, and the scope of surgery. The results showed that the preoperative HAMD score $(\mathrm{Y})$ was in negative correlation with the length of education $\left(\mathrm{X}_{1}\right)$ and positive correlation with the main symptoms $\left(\mathrm{X}_{2}\right)$, and had no significant correlation with other factors. According to the regression equation, $\mathrm{Y}=$ $-0.4641 \mathrm{X}_{1}+0.2867 \mathrm{X}_{2}(\mathrm{P}<0.05)$. The postoperative HAMD score $(\mathrm{Y})$ was in positive correlation with the parity $\left(\mathrm{X}_{1}\right)$ and the presence of medical complications $\left(\mathrm{X}_{2}\right)$ and negative correlation with the age $\left(\mathrm{X}_{3}\right)$, and had no significant correlation with other factors. According to the regression equation, $\mathrm{Y}=0.3753 \mathrm{X}_{1}+0.1975 \mathrm{X}_{2}-0.2975 \mathrm{X}_{3}(\mathrm{P}<0.05)$.

\subsection{SCL-90 Scores Before and After Surgery}

According to the comparison of SCL-90 scores before and after hysterectomy, the number of positive items and the somatic factor scores were generally significantly lower after hysterectomy, and compulsion, depression, anxiety, hostility, panic, paranoid, psychosis factor scores, etc. also decreased significantly $(\mathrm{P}<0.01)$. Patients of different age groups showed different extents of change. The trends of change for the group of patients aged between 40 and 45 years old and the group of patients aged between 45 and 50 years old were relatively obvious, and the postoperative ratings of the other two groups had many decreased items compared with the preoperative levels, but very few of these items had statistically significant differences (Table 4).

Table 4. SCL-90 scores before and after surgery.

\begin{tabular}{|c|c|c|c|c|c|c|}
\hline \multirow{2}{*}{ Item } & \multicolumn{2}{|l|}{$<40$} & \multicolumn{2}{|l|}{$40 \sim<45$} & \multicolumn{2}{|l|}{$45 \sim<50$} \\
\hline & Before & After & Before & After & Before & After \\
\hline Total score & $32.15 \pm 9.42$ & $27.31 \pm 8.49$ & $48.54 \pm 7.56$ & $29.37 \pm 8.47 * *$ & $44.58 \pm 9.18 * *$ & $29.45 \pm 9.46$ \\
\hline Positive list & $23.47 \pm 7.52$ & $18.64 \pm 8.48$ & $30.25 \pm 9.96$ & $16.72 \pm 8.76^{* *}$ & $27.41 \pm 9.54 * *$ & $19.48 \pm 9.98$ \\
\hline Somatic & $0.41 \pm 0.28$ & $0.40 \pm 0.34$ & $0.74 \pm 0.36$ & $0.35 \pm 0.32 * *$ & $0.82 \pm 0.53$ & $0.49 \pm 0.35 *$ \\
\hline Compulsory & $0.39 \pm 0.25$ & $0.34 \pm 0.30$ & $0.72 \pm 0.42$ & $0.34 \pm 0.31 * *$ & $0.62 \pm 0.36$ & $0.59 \pm 0.45$ \\
\hline Sensitivity & $0.37 \pm 0.23$ & $0.36 \pm 0.32$ & $0.64 \pm 0.52$ & $0.29 \pm 0.24 * *$ & $0.26 \pm 0.24$ & $0.24 \pm 0.21$ \\
\hline Depression & $0.47 \pm 0.32$ & $0.42 \pm 0.35$ & $0.68 \pm 0.59$ & $0.24 \pm 0.24^{* *}$ & $0.34 \pm 0.30$ & $0.32 \pm 0.31$ \\
\hline Anxiety & $0.34 \pm 0.28$ & $0.34 \pm 0.24$ & $0.51 \pm 0.36$ & $0.31 \pm 0.36^{*}$ & $0.51 \pm 0.36$ & $0.34 \pm 0.31 * *$ \\
\hline Hostility & $0.27 \pm 0.19$ & $0.24 \pm 0.20$ & $0.49 \pm 0.36$ & $0.30 \pm 0.29 *$ & $0.41 \pm 0.36$ & $0.29 \pm 0.28 *$ \\
\hline Panic & $0.43 \pm 0.34$ & $0.39 \pm 0.37$ & $0.40 \pm 0.25$ & $0.29 \pm 0.28 *$ & $0.34 \pm 0.32$ & $0.20 \pm 0.18^{*}$ \\
\hline Paranoid & $0.45 \pm 0.23$ & $0.43 \pm 0.38$ & $0.25 \pm 0.22$ & $0.21 \pm 0.19$ & $0.24 \pm 0.21$ & $0.21 \pm 0.19$ \\
\hline Neurotic & $0.39 \pm 0.27$ & $0.37 \pm 0.34$ & $0.31 \pm 0.25$ & $0.11 \pm 0.08 * *$ & $0.31 \pm 0.28$ & $0.24 \pm 0.21$ \\
\hline Others & $0.41 \pm 0.34$ & $0.44 \pm 0.32$ & $0.61 \pm 0.45$ & $0.20 \pm 0.18^{* *}$ & $0.52 \pm 0.33$ & $0.31 \pm 0.26^{*}$ \\
\hline
\end{tabular}

Table 4. Continued.

\begin{tabular}{|c|c|c|c|c|}
\hline \multirow{2}{*}{ Item } & \multicolumn{2}{|l|}{$>50$} & \multicolumn{2}{|l|}{ Total } \\
\hline & Before & After & Before & After \\
\hline Total score & $30.14 \pm 8.14$ & $19.48 \pm 8.99 *$ & $41.25 \pm 9.77$ & $27.16 \pm 8.81 * *$ \\
\hline Positive list & $21.58 \pm 8.45$ & $19.87 \pm 9.82$ & $26.41 \pm 9.12$ & $17.45 \pm 7.49 * *$ \\
\hline Somatic & $0.46 \pm 0.38$ & $0.38 \pm 0.34$ & $0.61 \pm 0.58$ & $0.43 \pm 0.38 * *$ \\
\hline Compulsory & $0.51 \pm 0.31$ & $0.48 \pm 0.34$ & $0.68 \pm 0.36$ & $0.34 \pm 0.25 * *$ \\
\hline
\end{tabular}




\begin{tabular}{lllll}
\hline \multirow{2}{*}{ Item } & $>\mathbf{5 0}$ & & Total & After \\
\cline { 2 - 5 } & Before & After & Before & $0.29 \pm 0.36^{* *}$ \\
\hline Sensitivity & $0.42 \pm 0.31$ & $0.28 \pm 0.21^{*}$ & $0.64 \pm 0.25$ & $0.24 \pm 0.23^{* *}$ \\
Depression & $0.38 \pm 0.32$ & $0.24 \pm 0.21^{*}$ & $0.58 \pm 0.35$ & $0.24 \pm 0.15^{* *}$ \\
Anxiety & $0.45 \pm 0.37$ & $0.38 \pm 0.34$ & $0.47 \pm 0.36$ & $0.34 \pm 0.31^{* *}$ \\
Hostility & $0.34 \pm 0.21$ & $0.31 \pm 0.25$ & $0.61 \pm 0.36$ & $0.36 \pm 0.21^{* *}$ \\
Panic & $0.29 \pm 0.21$ & $0.24 \pm 0.21$ & $0.58 \pm 0.32$ & $0.22 \pm 0.19$ \\
Paranoid & $0.18 \pm 0.16$ & $0.14 \pm 0.12$ & $0.24 \pm 0.21$ & $0.34 \pm 0.32^{* *}$ \\
Neurotic & $0.24 \pm 0.21$ & $0.22 \pm 0.19$ & $0.54 \pm 0.36$ & $0.39 \pm 0.34^{* *}$ \\
Others & $0.52 \pm 0.41$ & $0.48 \pm 0.37$ & $0.61 \pm 0.44$ & \\
\hline
\end{tabular}

Compared with the results before surgery, ${ }^{*} \mathrm{P}<0.05,{ }^{* *} \mathrm{P}<0.01$.

\subsection{SRHMS Results of Differently Aged Patients}

The SRHMS showed no significant difference among patients of different ages ( $\mathrm{P}>0.05$ for all). Nevertheless, the physical and overall health levels tended to decline with the increase of age, and the psychological, and social health levels showed a V-shaped trend (Table 5).

Table 5. SRHMS scores of differently aged patients.

\begin{tabular}{llll}
\hline Age & $<\mathbf{4 0}$ & $\mathbf{4 0 ~ < 4 5}$ & $\mathbf{4 5 ~ < 5 0}$ \\
\hline Physiological health & $140.31 \pm 34.12$ & $142.36 \pm 48.69$ & $141.37 \pm 42.36$ \\
Psychological health & $113.58 \pm 24.69$ & $105.36 \pm 65.35$ & $106.34 \pm 42.65$ \\
Social health & $89.24 \pm 24.66$ & $82.34 \pm 31.26$ & $81.91 \pm 24.69$ \\
Total score & $334.91 \pm 84.25$ & $339.24 \pm 139.14$ & $334.85 \pm 106.59$ \\
\hline
\end{tabular}

\subsection{Correlation Between Self-Concept and SCL-90 and SRHMS Scores}

Except for a reverse factor (self-criticism), all the eight factors of the Self-Concept Scale and total score thereof were in significant negative correlation with the SCL-90 total score and all its factors $(\mathrm{P}<0.01)$, and in significant positive correlation with the total score, sub-scale scores and all factors of the SRHMS $(\mathrm{P}<0.05)$. Correlation between the self-concept and SCL-90 total score, SRMHS total score and its sub-scale scores (Table 6).

Table 6. Correlation between self-concept and SCL-90 and SRHMS scores.

\begin{tabular}{|c|c|c|c|c|c|}
\hline \multirow{2}{*}{ Self-concept } & \multirow{2}{*}{ SCL-90 score } & \multicolumn{4}{|l|}{ SRHMS } \\
\hline & & Physiological health & Psychological health & Social health & Scale score \\
\hline Self-identity & $-0.42 * *$ & $0.31 * *$ & $0.38 * *$ & $0.35 * *$ & $0.41 * *$ \\
\hline Self-satisfaction & $-0.36 * *$ & $0.21 * *$ & $0.34 *$ & $0.36^{*}$ & $0.36^{* *}$ \\
\hline Self-action & $-0.48 * *$ & $0.26^{*}$ & $0.35 *$ & $0.28 * *$ & $0.28^{*}$ \\
\hline Physical self & $-0.46^{* *}$ & $0.37 * *$ & $0.26 * *$ & $0.42 * *$ & $0.34 * *$ \\
\hline Ethical self & $-0.42 * *$ & $0.18 * *$ & $0.37 * *$ & $0.40^{*}$ & $0.34 * *$ \\
\hline Psychological self & $-0.38 * *$ & $0.25^{*}$ & $0.29 *$ & $0.29 *$ & $0.29 * *$ \\
\hline Family self & $-0.41 * *$ & $0.22 * *$ & $0.41 * *$ & $0.34 * *$ & $0.28 * *$ \\
\hline Social self & $-0.46 * *$ & $0.30 * *$ & $0.36 * *$ & $0.37 *$ & $0.41 *$ \\
\hline Total score & $-0.45 * *$ & $0.24 *$ & $0.33 *$ & $0.35 * *$ & $0.30 * *$ \\
\hline Self-criticism & 0.06 & 0.03 & 0.01 & -0.06 & -0.02 \\
\hline
\end{tabular}

$* \mathrm{P}<0.05, * * \mathrm{P}<0.01$

\section{Discussion}

Many issues in the field of obstetrics and gynecology are related with the psychosomatic medicine. According to epidemiological surveys, when perplexed by menorrhagia and/or dysmenorrhea, premenstrual tension and other gynecological symptoms, nearly half of premenopausal women are so much affected physically and psychologically by the diseases that they would feel nervous, depressed, annoyed and distressed, and may have psychiatric symptoms such as irritability, crying, anxiety, depression, etc. These diseases can considerably affect the quality of life and living conditions of the patients, and may even lead to social dysfunction [9, 10]. Hysterectomy is the primary treatment method for these diseases [11]. With the development of psychosomatic medicine, people have begun to pay attention to the change in psychological status of the patients who have undergone the hysterectomy and lost their reproductive organs. Cooper discovered in his early studies that more than half of the postoperative patients would exhibit symptoms such as fatigue, insomnia, depression, sexual dysfunction, etc. He believed that these symptoms were caused by the hysterectomy and named them as post-hysterectomy syndrome [12]. However, this idea was disproved by a large number of prospective studies conducted afterwards. It was found in the studies that the hysterectomy can recover the daily activities, work, social intercourse, and sexual activities while removing the diseases and is thus beneficial to the psychiatric and somatic health of the patients [13]. In this study, it is revealed in the self-rating and nurse-administered 
rating results on depression before and after the hysterectomy that the patients had drastically decreased scores and most patients had no or apparently relieved depression after the hysterectomy. The postoperative incidence rate of depression was significantly lower than the preoperative rate and the incidence rate of moderate to severe depression decreased from $48.98 \%$ to $13.54 \%$ after hysterectomy. The number of positive items and the somatic factor scores of the postoperative SCL-90 were decreased significantly after hysterectomy. Meanwhile, the total score and scores of compulsion, depression, anxiety, hostility, paranoid and psychosis factors also decreased significantly after hysterectomy. This suggests that while affecting the physical health of the patients, the diseases also perplex the patients psychologically, leading to severe depressive tendencies and other psychiatric symptoms. The hysterectomy removes the factors that affect the psychological health of the patients as well as treating their somatic diseases. Therefore, the depression is relieved after the hysterectomy with improved psychological status and the hysterectomy is beneficial to the psychosomatic health of the patients [14].

Good interpersonal relationships play an important part on the psychological health of the patients. Women are generally more sensitive to changes of interpersonal relationships [15]. Women are also physiologically particular in that the endocrine changes can easily lead to fluctuating emotions and decreased emotional behavior and regulatory ability. In addition, due to factors in the custom and tradition, women have to bear more responsibility to take care of the family. They would have tensions in interpersonal relationships when they are ill or under overwhelming pressure from the work and have change in the body, emotions, attitudes, and behaviors. They may have interpersonal conflicts and lose their psychological balance [16] As can be seen from relevant analysis, improving the interpersonal relationships is an effective method to boost the psychological self-concept of the patients [17]. Therefore, the healthy development of the patients themselves is very important for the prevention of interpersonal barriers and the improvement of interpersonal relationships [18].

Patients in the present study had self-rating and nurse-administered rating depression scores positively correlated with the preoperative levels. The preoperative HAMD score is inversely proportional to the length of education and directly proportional to the main symptoms. The postoperative HAMD score is in positive correlation with the parity and the medical complications and in negative correlation with the age. These results indicate that the postoperative depression levels of the patients in the present study was affected by the preoperative levels, and patients who were susceptible to moderate to severe depression before and after the hysterectomy included those with lower level of education, younger age, higher parity, dysmenorrhea complaints, and medical complications. The change of the postoperative psychological status can be predicted by assessing the preoperative psychological status, and there is remarkable consistency between the psychological self-rating and nurse-administered rating results. Therefore, the medical staff can take advantage of the psychological self-rating data of the patients to conduct early psychological counseling and intervention before and after the hysterectomy for patients highly susceptible to psychiatric disorders so as to reduce the incidence of perioperative psychiatric disorders [19].

Patients in different age groups of the present study showed different extents of change in various SCL-90 factor scores before and after the hysterectomy. The group of patients aged between 40 and 45 years showed change before and after the hysterectomy generally the same as the overall change. Although the groups of patients aged less than 40 years and above 50 years had generally reduced postoperative SCL-90 factor scores when compared with the preoperative levels, there were very few items with significant difference, which indicated poorer psychiatric health conditions in female patients in the two groups than those in the group aged between 40 and 45 years. There are a few possible reasons for this result. 1) The uterus has its special social, sexual, and psychological roles. For young and less-educated women, the uterus does not only perform its social function as an organ to nurture the next generation, but is also a symbol of their identity and sexual attraction. When they have to undergo hysterectomy, they would feel depressed and be perplexed by postoperative psychological maladjustment for their loss of reproductive function and sexual organs [20]. 2) Women aged 40-50 years old exposed to heavy social and family responsibilities would feel aggravated mental burdens when they are perplexed by illnesses. Hysterectomy can release them from the pain of illnesses and enable them to fulfill a variety of social tasks with enormous energy. Therefore, the postoperative psychological status of the patients will be improved significantly [21]. 3) In addition to gynecological diseases, patients over the age of 50 patients are also victims of various medical complications, such as coronary heart disease, hypertension, diabetes, etc. These disease are more often than not complicated with psychological maladjustment, and hysterectomy cannot help mitigate these complications or relieve the psychological maladjustment caused these diseases. Therefore, there was little change found in some factor scores in the psychological assessment in patients aged 50 years or higher [22].

Some risk factors that can easily affect the perioperative psychological status of the patients before and after hysterectomy are found in the present study. Particularly, the group of patients highly susceptible to perioperative depression is identified. We wish our clinical staff would pay more attention to the psychological health of the patients to better ensure the perioperative psychosomatic health of the patients while treating the somatic diseases of the patients.

\section{References}

[1] Okunlola MA. Umuerri C. Pattern of mental ill health morbidities following hysterectomy for benign gynaecological disorders among Nigerian women [J]. Int J Ment Health Syst, 2009, 3 (1): 18-20. 
[2] Marvan ML. Catillo-Lopez RL. Psychological meaning of a woman with a hysterectomy among Mexican physicians and women [J]. Women Health, 2012, 52 (7): 658-78.

[3] Wong LP. Arumugam K. et al. Physical, psychological and sexual effects in multi-ethnic Malaysian women who have undergone hysterectomy [J]. J Obstet Gynaecol Res, 2012, 38 (8): 1095-105.

[4] Shehmar M. Gupta JK. et al. The influence of psychological factors on recovery from hysterectomy [J]. J R Soc Med, 2010, 103 (2): 56-9.

[5] Brouwer D, Meijer RR. On the Factor Structure of the Beck Depression Inventory-II: G Is the Key [J]. Psychol Assess, 2012, 7 (16): 334-38.

[6] Zimmerman M, Martinez J. Symptom differences between depressed outpatients who are in remission according to the Hamilton Depression Rating Scale who do and do not consider themselves to be in remission [J]. 2012, 142 (3): 77-81.

[7] Russinova Z. Rogers ES. Conceptualization and measurement of mental health providers' recovery-promoting competence: The Recovery Promoting Relationships Scale (RPRS) [J]. Psychiatr Rehabil J, 2013, 36 (1): 7-14.

[8] Esplen MJ. Stuckless N. et al. The FAP self-concept scale (adult form) [J]. Fam Cancer, 2009, 8 (1): 39-50.

[9] Oppermann K. Fuchs SC. et al. Physical, psychological, and menopause-related symptoms and minor psychiatric disorders in a community-based sample of Brazilian premenopausal, perimenopausal, and postmenopausal women [J]. Menopause, 2012, 19 (3): 355-60.

[10] Bauld R. Brown RF. et al. Stress, psychological distress, psychosocial factors, menopause symptoms and physical health in women [J]. Maturitas, 2009, 62 (2): 160-5.

[11] Wuntakal R. Erskine K. et al. Subtotal hysterectomy and possible psychological benefits with regards to keeping the cervix in afrocarribean women [J]. BJOG, 2009, 118 (6): 1137-8.

[12] Cooper R. Mishra G. et al. Hysterectomy and subsequent psychological health: findings from a British birth cohort study [J]. J Affect Disord, 2009, 115 (1): 122-30.
[13] Yen JY. Chen YH. et al. Risk factors for major depressive disorder and the psychological impact of hysterectomy: a prospective investigation [J]. Psychosomatics, 2008, 49 (2): 137-42.

[14] Persson P. Brynhildsen J. et al. A 1-year follow up of psychological wellbeing after subtotal and total hysterectomy--a randomised study [J]. BJOG, 2010, 117 (4): 479-87.

[15] Izydorczyk B. Psychological profile of bodily self features of young Polish women--comparative analysis of bodily self structure of woman with eating disorders and psychosis [J]. Psychiatr Pol, 2011, 45 (5): 653-70.

[16] Garcia-Arroyo JM. Dominguez-Lopez ML. et al. Psychological study of the dysthymic disorder in the woman $[\mathrm{J}]$. Actas Esp Psiquiatr, 2011, 39 (5): 331-3.

[17] Moliver N. Mika E. et al. Yoga experience as a predictor of psychological wellness in women over 45 years [J]. Int J Yoga, 2013, 6 (1): 11-9.

[18] Molina KM. Alcantara C. et al. Household structure, family ties, and psychological distress among U.S.-born and immigrant Latino women [J]. J Fam Psychol, 2013, 27 (1): 147-58.

[19] Lachmi-Epstein A. Mazor M. et al. Psychological and mental aspects and "tender loving care" among women with recurrent pregnancy losses [J]. Harefuah, 2012, 151 (11): 633-7.

[20] Bjelanovic V. Babic D. et al. Pathological pregnancy and psychological symptoms in women [J]. Coll Antropol, 2012, 36 (3): 847-52.

[21] Whang W. Davidson KW. et al. Global Psychological Distress and Risk of Atrial Fibrillation Among Women: The Women's Health Study [J]. J Am Heart Assoc, 2012, 1 (3): 107-9.

[22] Lustyk MK. Douglas HA. et al. Hemodynamic and psychological responses to laboratory stressors in women: assessing the roles of menstrual cycle phase, premenstrual symptomatology, and sleep characteristics [J]. Int J Psychophysiol, 2012, 86 (3): 283-90. 